\title{
FAMILIA CASSIDULINIDAE (PROTOZOA: FORAMINIFERIDA) EN CANALES Y FIORDOS PATAGÓNICOS CHILENOS
}

\author{
CASSIDULINIDAE FAMILY (PROTOZOA: FORAMINIFERIDA) FROM \\ CHILEAN PATAGONIC CHANNELS AND FJORDS
}

Francisco Arellano ${ }^{1}$, Lyta Quezada ${ }^{1} \&$ Carlos Olave ${ }^{2}$

\begin{abstract}
A total of 164 marine superficial sediments samples were collected in the channels and fjords area of Chile $\left(42^{\circ}-56^{\circ} \mathrm{S}\right)$ in order to investigate the presence of the Cassidulinidae family. (Protozoa: Foraminiferida). It was identified 12 species, classified in 4 genera. The most wide geographic distribution taxa in the channels and fjords were: Globocassidulina rossensis (50,6\% of samples), Cassidulinoides parkerianus $(42,1 \%)$ and Cassidulina laevigata (34,8\%). Mention for presence of this 12 species from their localities in Chile was carried up. It was collected 29.033 specimens and the most abundant species were: Globocassidulina minuta (42,2 \%), Globocassidulina subglobosa (19,9\%) y Globocassidulina rossensis (16,7 \%).
\end{abstract}

Key words: Cassidulinidae, Chile, biodiversity, geographic and bathymetric distribution.

\section{RESUMEN}

Se revisó 164 muestras de sedimento marino superficial recolectadas en el área de canales y fiordos patagónicos chilenos $\left(42^{\circ}-56^{\circ} \mathrm{S}\right)$ para investigar la presencia de la Familia Cassidulinidae (Protozoa: Foraminiferida). Se identificaron 12 especies, las que fueron clasificadas en 4 géneros. Los taxa con más amplia distribución geográfica en el área fueron Globocassidulina rossensis (50,6 \% de las muestras), Cassidulinoides parkerianus (42,1\%) y Cassidulina laevigata (34,8\%). Se indica la presencia de estas especies, en otras localidades de Chile. En un total de 29.033 ejemplares, las especies más abundantes fueron: Globocassidulina minuta (42,2\%), Globocassidulina subglobosa (19,9\%) y Globocassidulina rossensis (16,7 \%).

Palabras clave: Cassidulinidae, Chile, biodiversidad, distribución geográfica y batimétrica.

1 Laboratorio de Micropaleontología, Instituto de la Patagonia, Universidad de Magallanes, Casilla 113-D, Punta Arenas, Chile.

2 Fundación Centro de Estudios del Cuaternario Fuego Patagonia y Antártica (CEQUA). Avda. 21 de Mayo 1690, Punta Arenas, Chile. 


\section{INTRODUCCIÓN}

Las aguas chilenas se clasifican en aguas oceánicas, continentales, insulares y antárticas ya que se considera que el espacio marítimo chileno abarca, el Mar Territorial que comprende las 12 millas cercanas a la costa, la zona económica exclusiva hasta aproximadamente las 200 millas en los continentes americanos y antárticos y finalmente los alrededores de la isla de Pascua y Salas y Gómez (Inst. Geográfico Militar, 1993).

Este enorme y diverso ambiente acuático alberga una rica vida marina siendo los foraminíferos, uno de los grupos más frecuentes y abundantes en todos los ambientes marinos (Murray 1991). Sin embargo, en Chile han recibido poca atención, exceptuando la zona de canales y fiordos, que durante las tres últimas décadas, se ha investigado más sistemáticamente (Marchant 1993; Hromic 19963 19974 19985 , 1996, 2001; 2009 a y b, 2011 a, b y c; Hromic \& Zúñiga 2005, Zapata \& Moyano 1996, 1997, Violanti et al. 2000, entre otros). En dichos estudios se ha puesto en evidencia que los representantes de la Familia Cassidulinidae d'Orbigny 1839 , aparecen regularmente mencionados presentando una distribución amplia y una abundancia del $9,2 \%$ del total de los foraminíferos recolectados (Hromic, 20106).

La Familia Cassidulinidae con sus 20 géneros (Loeblich \& Tappan 1988) incluye formas con caparazones calcáreos, pequeños, lenticulares, subglobosos ligeramente alargados; planispirales, involutos, con abertura alargada en la periferia de la última cámara. Muchas de sus especies son cosmopolitas, mientras que unas pocas tienen distribuciones restringidas.

3 Hromic, T. 1996. Foraminíferos bentónicos de Campos de Hielo Sur, Parte 1: Canales Baker y Messier. Actas Jornadas Ciencias del Mar, Concepción, Chile.

4 Hromic, T. 1997 Análisis taxonómico y distribución de los foraminíferos bentónicos del Estrecho de Magallanes, extraídos durante la campaña Joint Magellan "VICTOR HENSEN" (1994) y su relación con la microfauna antártica. Actas IBMANT 97, Seminario Taller Internacional A. Wegener Institute (AWI) y Universidad de Magallanes, Punta Arenas, Chile.

5 Hromic, T. 1998 Foraminíferos y Biodiversidad. XVIII Congreso de Ciencias del Mar, Iquique, Chile. Actas de Resúmenes.

6 Hromic, T. 2010 Benthic Foraminifera of fjord and channels of southern Chile. International Congress "Forams 2010", Universidad de Bonn, Alemania.
El registro fósil indica que los cassidulinoídeos se encuentran a partir del Paleoceno (Loeblich \& Tappan 1988, Brady, 1884). En la cuenca austral chilena, se han hallado ejemplares fósiles de Cassidulina brocha Poag 1966 desde el piso Gaviotiano inferior al Miradoriano (Cañón \& Ernst 1974, In: Natland et al, 1974), de edad Eoceno y en la formación Brush Lake (Hromic 1991) de edad Mioceno. En el sur de Chile, se ha encontrado: Globocassidulina crassa (d'Orbigny) y Cassidulinoides parkerianus (Brady) en la Formación Tubul (Martínez \& Osorio 1968) y Caleta Huetón, Arauco (Martínez 1976). Globoscassidulina subglobosa (Brady) fue encontrada en los estratos de Pupunahue (Marchant 1990), en la Formación Tubul (Martínez \& Osorio 1968) y en San Sebastián (Martínez \& Parada 1968). En esta misma formación también hay registros del género Ehrenbergina: Ehrenbergina notoensis Asano (Martínez \& Parada 1968).

En el presente, se sabe de la presencia de 37 especies en los mares chilenos (Boltovskoy \& Theyer 1970, Ingle et al. 1980 Zapata \& Moyano 1997, Figueroa et al. 2005 entre otros).

Según Murray (1991) los géneros Cassidulina y Globocassidulina son infaunales, de vida libre, se les encuentra en sustratos lodosos y arenosos, detritívoros y marinos. Habitan en aguas temperado-frías, tanto en la zona de plataforma abierta (100-200 m) como en la zona batial, prefiriendo las especies de Globocassidulina profundidades $>200 \mathrm{~m}$. Ehrenbergina tendría características similares: infaunal, de vida libre, preferentemente habitando en sustratos lodosos, detritívora, marina, propia de aguas frías y viviendo en plataforma externa y zona batial. No se registra en la literatura más antecedentes para otras especies o géneros.

En el presente trabajo se da a conocer la lista de especies de la Familia Cassidulinidae presentes en canales y fiordos patagónicos de Chile $\left(42^{\circ}\right.$ y $\left.56^{\circ} \mathrm{S}\right)$, su abundancia rango latitudinal y batimétrico.

\section{MATERIAL Y MÉTODOS}

El material utilizado en el presente estudio corresponde a 164 muestras de sedimento marino superficial recolectadas en un total de 8 expediciones, los cruceros científicos CIMAR FIORDOS 2 , 
Tabla 1. Expedición, fecha y localidad visitada.

\begin{tabular}{|c|c|c|c|}
\hline Expedición* & Fecha & Destino & № muestras \\
\hline CIMAR 2 FIORDOS & $\begin{array}{c}14 \text { octubre }-9 \\
\text { noviembre } 1996\end{array}$ & Golfo de Penas y el estrecho de Magallanes. & 36 \\
\hline CIMAR 3 FIORDOS & 6 - 18 octubre 1997 & $\begin{array}{l}\text { Zona del estrecho de Magallanes (y canales australes), } \\
\text { Canal Beagle y Cabo de Hornos }\end{array}$ & 31 \\
\hline CIMAR 8 FIORDOS & $1-26$ julio 2002 & XI Región (Aysén) y sus canales & 17 \\
\hline CIMAR 10 FIORDOS & $\begin{array}{c}17 \text { agosto }-3 \\
\text { septiembre } 2004\end{array}$ & $\begin{array}{l}\text { Entre Puerto Montt y Boca del Guafo (Norpatagonia } \\
\text { chilena), X Región de Chile }\end{array}$ & 19 \\
\hline CIMAR 11 FIORDOS & $16-27$ julio 2005 & X Región de Chile y fiordos del mar interior de Chiloé & 17 \\
\hline CIMAR 12 FIORDOS & 4 - 24 julio 2006 & $\begin{array}{l}\text { Canales y fiordos de la X Región (entre Puerto Montt y } \\
\text { boca del Guafo) }\end{array}$ & 15 \\
\hline CIMAR 13 FIORDOS & 21 julio - 11 agosto 2007 & Boca del Guafo y golfo Elefantes, incluido el fiordo Aysén & 13 \\
\hline CIMAR 14 FIORDOS & $\begin{array}{c}27 \text { octubre }-26 \\
\text { noviembre } 2008\end{array}$ & $\begin{array}{l}\text { Entre golfo de Penas a canal Trinidad, incluido el canal } \\
\text { Baker }\end{array}$ & 16 \\
\hline
\end{tabular}

* Detalles localidades muestreo: Informes preliminares (CONA, Chile) www.cona.cl

$3,8,10,11,12,13$ y 14 . Dichos cruceros fueron organizados por la Armada de Chile, (Comité Oceanográfico Nacional, Chile), entre 1996 y 2008 y se concretaron en la zona de canales patagónicos $\left(42^{\circ}\right.$ y $56^{\circ}$ S) (Tabla 1). El procesamiento de las muestras siguió las indicaciones de Boltovskoy (1965). Las muestras se lavaron utilizando un tamiz de 63 micrones y luego se secaron. Posteriormente se apartó 50 gramos cuando fue posible para la extracción total de los foraminíferos. Los ejemplares se separaron uno a uno bajo lupa binocular y fueron depositados en reglillas portaforaminíferos. La clasificación a nivel genérico siguió a Loeblich \& Tappan (1988) y Decrouez (1989) y a nivel específico, el catálogo de Ellis \& Mesina (1944 et seq.) y diversos autores que han publicado sobre foraminíferos del cono sur de América (Brady 1884, Earland 1933, 1934, Barker 1960, Boltovskoy et al. 1980, Zapata \& Moyano 1996, entre otros).

Los ejemplares se encuentran depositados en la colección del Instituto de la Patagonia, Universidad de Magallanes.

Las abundancias absolutas de foraminíferos se transformaron a ejemplares por gramo, debido a la gran diferencia de peso de las muestras tras el lavado. Las citas para Chile se obtuvo de la revisión de los trabajos publicados sobre foraminíferos para aguas chilenas.

\section{RESULTADOS}

Sistemática

En total se recolectaron 29.033 ejemplares de la Familia Cassidulinidae los que se distribuyeron en 4 géneros y 12 especies, quedando una de ellas con nomenclatura abierta. El género Cassidulina (6,3\% de los ejemplares) aportó con dos especies, Cassidulinoides (11,8\% de los ejemplares) con 4 especies; Ehrenbergina (1,5\% de ejemplares) con una especie y Globocassidulina $(80,5 \%$ de los ejemplares) con 5 especies (Tabla 2).

Descripción de las especies

\section{Género Cassidulina d'Orbigny 1826}

Cassidulina carinata (Cushman, 1922)

(Fig. 1 A; Fig. 4: 2a, b, c y d)

Sin.: Cassidulina laevigata (d'Orbigny) var. carinata Cushman 1922, 104: 124, pl. 2, figs. 6 7. Cushman \& Hugues 1925, (1), pt. 3: 52, pl. 8, figs. 11 - 12. Cushman \& Parker 1931: 14, pl. 2, figs. $14 \mathrm{a}, \mathrm{b}$.

Cassidulina carinata (Cushman) Murray 1991: 330. Hromic 2001: 144, St. B3. Zapata \& Cea 2004, 75: 19: pl. 3, fig. 5

Diagnosis: Caparazón lenticular, suavemente biconvexo, transparente, con poros finos, comprimido, con 4 o más cámaras visibles, en la última vuelta, 
Tabla 2. Lista taxonómica de la Familia Cassidulinidae, presente en fiordos australes (42ํㅡ - 56은 ).

Familia CASSIDULINIDAE d' Orbigny, 1839

Subfamilia Cassidulininae d' Orbigny, 1839

Género Cassidulina d' Orbigny, 1826 Eoceno Tardío- Holoceno

Cassidulina carinata (Cushman 1922)

Cassidulina laevigata (d' Orbigny, 1826)

Género Cassidulinoides Cushman, 1927 Pleistoceno- Holoceno

Cassidulinoides brady (Norman, 1881)

Cassidulinoides parkerianus (Brady, 1884)

Cassidulinoides parvus (Earland, 1934)

Cassidulinoides porrectus (Heron Allen \& Earland, 1932)

Género Globocassidulina Voloshinova, 1960 Eoceno Tardío- Holoceno

Globocassidulina crassa (d'Orbigny, 1839)

Globocassidulina minuta (Cushman, 1933)

Globocassidulina rossensis (Kennett, 1967)

Globocassidulina subglobosa (Brady, 1881)

Globocassidulina sp. 1

Subfamilia Ehrenbergininae Cushman 1927

Género Ehrenbergina Reuss 1850

Ehrenbergina pupa (d'Orbigny, 1839) Eoceno-Holoceno

con un reborde, delgado, carenado, muy variable, ligeramente agudo y ondulado hasta serrado. Las cámaras son planas, con suturas radiales, escasamente deprimidas. Apertura periférica alargada y estrecha, paralela al margen.

Observaciones: Esta especie suele confundirse con $C$. laevigata de la cual se distingue por la pronunciada carena, de aspecto aserrado que presenta C. carinata. Cosmopolita (Murray 1991).

Abundancia: 11,14 ejemplares /gramo.

Distribución latitudinal

Rango: $43^{\circ} 45^{\prime}-54^{\circ} 55^{\prime}$

Se encontró en 15 muestras: CIMAR 8 FIORDOS (St.: 65, 9, 54, 45, 61, 1y 78). CIMAR 3 FIORDOS (St.: 8, 9, 24, 28, 36, 40, 53 y 57). Se presenta con una distribución disyunta en la parte norte de la zona de los canales y fiordos con muy escasos ejemplares y en la zona de canales y fiordos australes con abundancias mayores.

Profundidad: entre 45 y $650 \mathrm{~m}$.

Cassidulina laevigata d' Orbigny, 1826

(Fig. 1 B; Fig. 4: 1a, b y c)

Sin.: Cassidulina laevigata d'Orbigny, 1826: 282, lám. 6, figs. 4, 5. Williamson 1858: 68, lám. 6, figs. 141 - 142. Parker \& Jones 1865: 377, lám. 17, fig. 64 a, b, c. Brady 1884: 428, pl. LIV, figs. 1 - 3; Heron- Allen \& Earland 1932: 357; Earland 1934: 135; Boltovskoy \& Theyer 1970: 316, lám.
1, fig. 25. Boltovskoy et al. 1980: 22, pl. 7, figs. 4 - 6; Zapata et al. 1995: 25, lám. 3, figs. 1 - 2. Hromic 1996: 75, lám. 3, fig. 2. Loeblich \& Tappan 1987: 504. lám. 555. Figs. 1-5. Cusminsky, 1992: 24 (1): 21; lám. 5, fig. 2. Mackensen \& Hald 1988, 18 (1): 16-24, pl. 1. Murray 1991: 330. Figueroa et al. 2005: 18.

Diagnosis: Caparazón semicircular, comprimido, lenticular, margen periférico entero, suavemente lobulado, agudo, incipiente carena. Cinco pares de cámaras, biseriales y planispirales, ligeramente alargadas y angostas, en la vuelta final. Paredes lisas, brillantes y perforadas, con grandes poros. Suturas paralelas, levemente arqueadas y algo deprimidas, especialmente en la cara apertural. Apertura, hendidura alargada, angosta y paralela a la periferia, con un labio que se extiende desde la base.

Observaciones. Se asocia a sustratos lodosos y es cosmopolita (Murray 1991). Cassidulina laevigata se diferencia de C. carinata por tener un borde agudo, sin llegar a formar una verdadera carena.

Abundancia: 131,43 ejemplares/ gramo

Distribución latitudinal

Rango: $41^{\circ} 33^{\prime}-55^{\circ} 40^{\prime}$.

Se encontró en 57 muestras: CIMAR 2 FIORDOS (St.: 89, 87, 85, 43, 47, 5, 17, 23, 24, $40,41,76,70,50,60,59,56,64,6,8,27,25$, 29, 36, 72, 71, 75 y 57). CIMAR 10 FIORDOS (St.: 37 y 49). CIMAR 8 FIORDOS (St.: 54, 4, 45, 61 

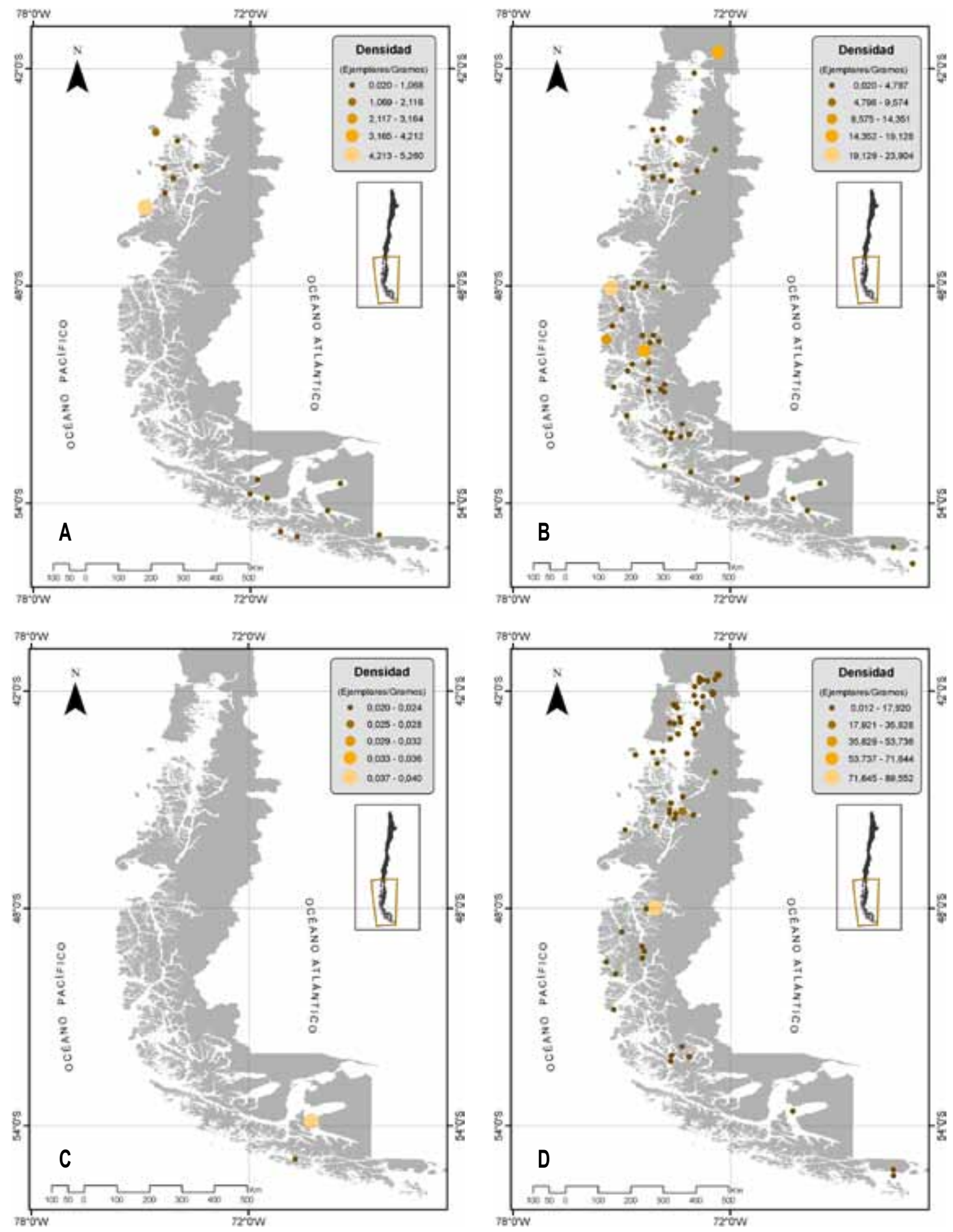

Fig. 1. Distribución geográfica y densidad en canales y fiordos patagónicos de: A.- Cassidulina carinata; B.- Cassidulina laevigata; C.- Cassidulinoides bradyi y D.- Cassidulinoides parkerianus. 
y 59). CIMAR 3 FIORDOS (St.: 8, 12, 16, 24, 34, 45, 53, 54 y 57). CIMAR 12 FIORDOS (St.: 7 y 16). CIMAR 13 FIORDOS (St.: 36, 37, 39, 41, 86 y 80). CIMAR 14 FIORDOS (St.: 29, 87, 15, 31 y 91). Presenta una distribución amplia en los canales y fiordos chilenos.

Profundidad: entre $45 \mathrm{~m}$ y $1.218 \mathrm{~m}$.

Género Cassidulinoides Cushman 1927

Cassidulinoides bradyi (Norman, 1881)

(Fig. $1 \mathrm{C}$ )

Sin.: Cassidulina bradyi Norman, 1881. Brady 1881, 21: 59. Brady 1884: 431, lám. 54, figs. 6 - 9. Cushman 1925, 1, 3: 52, pl. 8, figs. 3 - 5.

Cassidulinoides bradyi (Norman). Baker 1960, Sp. Pub. 9: 112; pl. 54, figs. 6 - 9. Ingle et al. 1980, 26 (2): 132. Mateu 1989. 2: 108. Murray 1991: 330.

Diagnosis: Caparazón oval, convexo, muy comprimido, alargado, con forma de riñón o de báculo; con un borde periférico delgado, afilado, puntiagudo o ligeramente redondeado. Los segmentos primarios son planispirales, los posteriores, oblicuos y alternados, biseriales. La apertura es pequeña, curva y se ubica en la cara interna de la cámara terminal.

Abundancia: 0,06 ejemplares /gramo

Distribución latitudinal

Rango: $53^{\circ} 52^{\prime}-54^{\circ} 55^{\prime}$

Se encontró en 2 muestras : CIMAR 3 (St.:

36 y 54). Constituye un nuevo registro para canales y fiordos.

Profundidad: entre $120 \mathrm{~m}$ y $280 \mathrm{~m}$

Cassidulinoides parkerianus (Brady, 1884)

(Fig. 1 D; Fig. 5: 3a y b)

Sin.: Cassidulina parkeriana Brady 1884: 432, lám. 54, figs. 11 - 16. Heron-Allen \& Earland 1932: 359, lám. 9, figs. 22 - 25.

Cassidulinoides parkerianus (Brady) Boltovskoy \& Theyer 1970: 318, lám. 2, fig. 2; Boltovskoy et al. 1980, 23, pl 8, figs. 1 - 4; Ward \& Webb 1986: 190, pl. 4, fig. 9; pl.8, fig. 6; Boltovskoy \& Totah 1987, 44; Zapata 1987, 56, lám. 5, figs. 1 y 2; Murray 1991: 330; Cusminsky 1992: 20, lám. 3, fig. 5. Marchant 1993, 57: lám. 1, figs. 3 a y b; Hromic 1996: 75, lám. 1, fig. 7; 1999: 94, fig. 3 y 9. Figueroa et al. 2005: 19
Diagnosis: Caparazón alargado, en forma de jota o bastón de diferente longitud; cámaras iniciales globosas y enrolladas, con tres a cuatro pares de cámaras rectas terminales, ligeramente infladas y perpendiculares al eje de crecimiento. Paredes finas, con perforaciones pequeñas y abundantes. Suturas nítidas, rectilíneas, deprimidas. Apertura terminal, ovalada, con un labio.

Observaciones: Especie cosmopolita (Zapata \& Moyano 1997), el ejemplar encontrado en los canales patagónicos posee un cuerpo más estilizado, transparente y las suturas se encuentran bastante nítidas.

Abundancia: 248,34 ejemplares /gramo.

Distribución latitudinal

Rango: $41^{\circ} 33^{\prime}-55^{\circ} 23^{\prime}$.

Se encontró en 69 muestras: CIMAR 11 FIORDOS (St.: 9, 33, 36, CQ2, CQ4, HC3, HC5, HC6, DQ2, DQ3, DQ4 y DQ5). CIMAR 2 FIORDOS (St.: $89,85,83,43,24,60,56,64,76,6$ y 57). CIMAR 10 FIORDOS (Estaciones: 2, 3, 7, 8, 17, 20, 22, 30, 35, 37, 39, 45 y 49). CIMAR 8 FIORDOS (St.: 11, 4, 14, 45, 61, 1, 78 y 71). CIMAR 3 FIORDOS (St.: 19, 34, 49 y 55). CIMAR 12 FIORDOS (St.: 8, 4, 5, 7, 16, 20, 33, 36 y QL9). CIMAR 13 FIORDOS (St.: 37, 41, 43, 84, 80, 77, 76 y 46). CIMAR 14 FIORDOS (St.: 23, 22, 95 y 7).

Profundidad: entre $5 \mathrm{~m}$ y $1.218 \mathrm{~m}$.

Cassidulinoides parvus (Earland, 1934)

(Fig. 2 A; Fig. 5: 1a y b)

Sin.: Ehrenbergina parva Earland 1934 (5): 139, pl. 6, figs. $28-32$.

Diagnosis. Caparazón biserial, 4 a 6 pares de cámaras infladas; primeras cámaras enrolladas hacia el lado ventral y las continuas, rectas; paredes gruesas, poros toscos, con apariencia granular, apertura, en cara interna de la cámara final. Suturas deprimidas. Con una protuberancia en el lado ventral de las cámaras más tempranas.

Observaciones: Se asemeja a Cassidulinoides parkerianus, sin embargo parvus posee cámaras más globosas, caídas, transparentes, bien formadas y orientadas de manera oblicua, las suturas están bien definidas.

Abundancia: 3,98 ejemplares/gramo

Distribución latitudinal

Rango: $52^{\circ} 41^{\prime}-55^{\circ} 40^{\prime}$ 

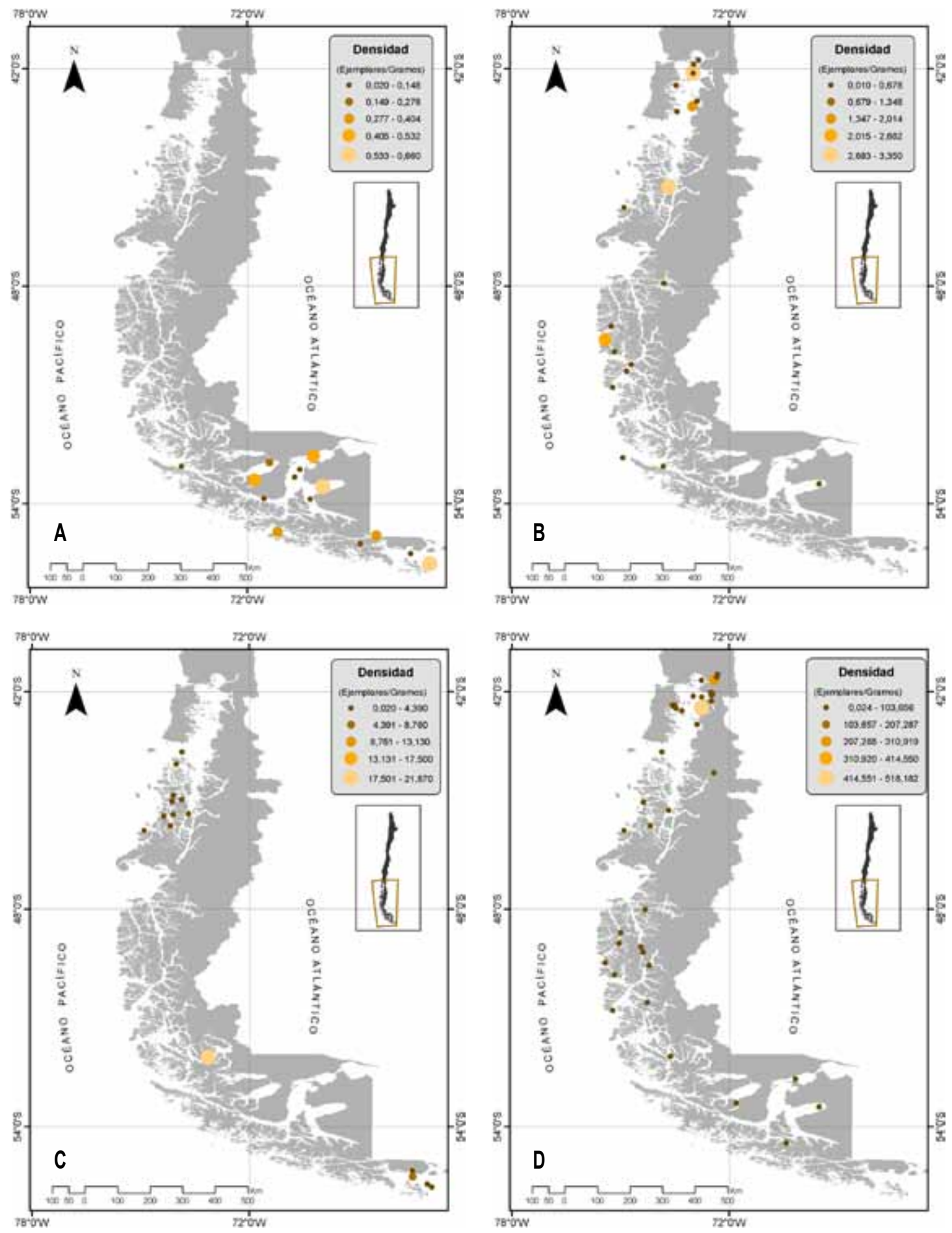

Fig. 2. Distribución geográfica y densidad en canales y fiordos patagónicos de: A.- Cassidulinoides parvus; B.- Cassidulinoides porrectus; C.- Globocassidulina crassa, y D.- Globocassidulina minuta. 
Se encontró en 16 muestras: CIMAR 3 FIORDOS St.: 4, 5, 6, 8, 12, 19, 20, 24, 28, 30, $40,45,49,50(3), 54,56$, en la zona austral de canales y fiordos

Profundidad: entre $41 \mathrm{~m}$ y $650 \mathrm{~m}$

Cassidulinoides porrectus (Heron Allen - Earland) (Fig. 2 B; Fig. 5: 4a y b)

Sin.: Cassidulina crassa d' Orbigny var. porrecta Heron Allen \& Earland, 1932, 4 : 358, lám. 9, figs. 34 - 37. Earland 1934, 10: 136. Boltovskoy et al. 1980, 22; pl. 7, figs. 1 - 3.

Cassidulina porrecta (Heron - Allen \& Earland) Boltovskoy 1964, 3 (3): 210, lám. 18, fig. 9;

Cassidulinoides porrectus (Heron Allen \& Earland ). Fillon 1974, 20 (2): 139 Ward \& Webb 1986: 190, pl. 8, figs.3, 7 - 10; pl. 11, figs. 1, 2;pl. 12 , figs. 1,3 .

Diagnosis: Caparazón alargado, aplanado, piriforme, biserial, cuatro pares de cámaras en la última vuelta, variables que tienen a alargarse. Suturas rectas, poco hendidas. Apertura perpendicular al borde, recta. Sin diente.

Observaciones: Esta especie es más aplanada, tiene las cámaras terminales alargadas y suturas menos visibles que G. crassa. La cámara final es similar a un trapezoide. Heron - Allen \& Earland (1932) atribuyen esta condición a formas seniles. Boltovskoy et al. (1980) señalan que puede encontrarse formas intermedias entre $C$. crassa y $C$. porrectus.

Abundancia: 14,24 ejemplares /gramo.

Distribución latitudinal

Rango: $41^{\circ} 45^{\prime}-53^{\circ} 27^{\prime}$

Se encontró en 22 muestras: CIMAR 11 FIORDOS (St.: 8, 9, 33, 36 y DQ5). CIMAR 2 FIORDOS (St.: 87, 83, 43, 40, 41 y 11). CIMAR 10 FIORDOS (St.: 16 y 39). CIMAR 8 FIORDOS (St.: 78); CIMAR 3 FIORDOS (St.: 12, 15, 30 y 57). CIMAR 12 FIORDOS (St.: 16). CIMAR 13 FIORDOS (St.: 43). CIMAR 14 FIORDOS (St.: 85 y 95).

Profundidad: entre 18 m y 970 m

Género Globocassidulina Voloshinova 1960

Globocassidulina crassa (d'Orbigny, 1839)

(Fig. 2C; Fig. 4: 4a y b)

Sin.: Cassidulina crassa d'Orbigny, 1839 b: 56 , lám. 7 , figs. 18 - 20; 1846: 213, lám. 21, figs. 42, 43. Brady 1884: 429, lám. 54, figs. 4, 5. Cushman \& Wickenden 1929: 12, lám. 5, fig. 5. Heron - Allen \& Earland 1932: 357, lám. 9 figs. 26 - 33; 358, (St. 388). Barker (1960) lám. 54. Figs. 4 - 5; Boltovskoy \& Theyer 1970: 315. pl. 3. fig. 1. Zapata \& Varela 1975: 15, lám. 1, fig. 4. Boltovskoy \& Totah 1987: 44. Murray 1991: 330. Zapata et al. 1995. Dimensiones 0,42 - 0,68 mm 41 S). Zapata \& Moyano 1996: 93.

Globocassidulina crassa Finger \& Lipps 1981: 117. Thompson 1978: 8 (3): 238, tb. 2; 252 , pl. 4. Violanti et al. 2000, 526, pl. 1 fig. 3. Páez et al. 2001: 133, pl. 2, fig. c. Zapata \& Cea 2004: 27, pl. 8., fig. 8.

Diagnosis: Caparazón circular ligeramente ovalado; lados suavemente convexos; margen periférico redondeado. Con cinco pares de cámaras, cortas, algo abultadas, la última rectangular o trapezoidal, lo que forma una esquina. Paredes finas, lisas, brillantes, nítidamente perforadas, con poros muy pequeños. Suturas levemente deprimidas. Apertura pequeña, ovalada, rodeada por un ancho reborde, situada perpendicularmente en la última cámara.

Observaciones: Violanti et al. 2000 menciona que esta especie es abundante y domina en el estrecho de Magallanes.

Abundancia: 36,95 ejemplares/gramo

Distribución latitudinal

Rango: $42^{\circ} 49^{\prime}-55^{\circ} 40^{\prime}$.

Se encontró en 15 muestras: CIMAR 2 FIORDOS (St.: 56). CIMAR 8 FIORDOS (St.: 65, 72, 4, $56,67,14,45,61,59$ y 78). CIMAR 3 FIORDOS (St.: 19, 32, 34 y 45).

Profundidad: entre $87 \mathrm{~m}$ y $274 \mathrm{~m}$

Globocassidulina minuta (Cushman, 1933)

(Fig. 2 D; Fig. 4: 5a y b)

Sin.: Cassidulina minuta Cushman 1933 a: 92, lám. 10, fig. 3 a y b. Boltovskoy et al. 1980, 22: pl. 7, figs. 7 - 11. Zapata \& Moyano 1997: 54, lám. 4, figs. 11 y 12. Murray 1991: 330. Marchant 1993: 64, lám. 1, figs. 2 a y b. Zapata et al. 1995. 92, lám. 10, fig. 3. Violanti et al. 2000, 521, pl. 1 , fig. 3.

Globocassidulina minuta (Cushman) Mateu 1989: 105, pl. 60, figs. 3 y 6. Zapata \& Olivares 2000: 59, pl. 3, fig. 10. Zapata \& Cea 2004: 27, lám. 8, fig. 9. 
Diagnosis: Caparazón pequeño, semicircular u oval, comprimido lateralmente; el margen periférico es redondo y lobulado. Con 4 pares de cámaras, levemente infladas, globosas y rectangulares, en la vuelta final. Sus paredes son lisas, brillantes y finamente perforadas. Suturas deprimidas, rectilíneas. La apertura es alargada, arqueada y angosta, paralela al margen periférico, posee un diente que nace en la sutura basal.

Observaciones: Se parece a G. crassa pero difiere de ella en que es más comprimida, posee 4 cámaras y la apertura es alargada, situada a lo largo de la base de la última cámara.

Abundancia: 953,95 ejemplares/gramo

Distribución latitudinal

Rango: $41^{\circ} 31^{\prime}-54^{\circ} 27^{\prime}$.

Se encontró en 42 muestras: CIMAR 11 FIORDOS (St.: 6, 7, HC3, HC5, HC6, HC8, DQ2, DQ3, DQ4 y DQ5). CIMAR 2 FIORDOS (St.: 83, 43, 76, 60 y 6). CIMAR 10 FIORDOS (St.: 3, 5, 6, 17, 22 y 25). CIMAR 8 Fiordos (St.: 72, 4, 63 y 78). CIMAR 3 FIORDOS (St.: 4, 24, 29, 30 y 57). CIMAR 12 FIORDOS (St.: 16 y 33). CIMAR 13 FIORDOS (St.: 37 y 43); CIMAR 14 FIORDOS (St.: 23, 85, 25, 22, 89, 87, 15 y 88).

Profundidad: entre $41 \mathrm{~m}$ y $700 \mathrm{~m}$

Globocassidulina rossensis (Kennett, 1967)

(Fig. 3 A; Fig. 4: 3a, b y c)

Sin.: Globocassidulina crassa (D'Orbigny) rossensis Kennett, 1967, 18(3) 134, pl. 11, figs. 4 - 6.

Globocassidulina crassa rossensis (Kennet, 1967) Fillon 1974: 138, pl. 1 figs. 1 - 7. Finger \& Lipps 1980: 177, pl.4, figs. 5 a, b y c. Cusminsky 1992, 24 (1): 21, lám. 5, fig. 4.

Cassidulina rossensis Kennett 1967 Boltovskoy et al. 1980: 22, pl. 7, figs. 15 - 17.

Globocassidulina rossensis (Kennett) Martínez \& Martínez-Pardo 1994: 1690, Tb. 1. Violanti et al. 2000: 521, pl. 1, fig. 4

Diagnosis: Caparazón con contorno subcircular, comprimido; cámaras infladas globosas, poligonales, 4 - 5 pares en la última vuelta; pared lisa, brillante y ligeramente perforada, blanca; suturas radiales, deprimidas, marcando las cámaras globosas. Apertura alargada y angosta, que termina con un claro reborde, generalmente con forma de $\mathrm{L}$, sobre la sutura basal, de la útima cámara, a veces se observa pequeño diente.
Abundancia: 374,58 ejemplares / gramo.

Distribución latitudinal

Rango: $41^{\circ} 33^{\prime}-55^{\circ} 27^{\prime}$.

Se encontró en 83 estaciones: CIMAR 11 FIORDOS (St.: 5, 6, 9, 33, 36 y CQ2). CIMAR 2 FIORDOS (St.: 91, 90, 89, 87, 85, 83, 42, 43, 47, 23, 24, 40, 41, 76, 70, 50, 59, 56, 64, 76b, 6, 28, $25,72,71,75,57$ y 58). CIMAR 10 FIORDOS (St.: $2,5,7,14,35,37,39$ y 49). CIMAR 8 FIORDOS (St.: 4, 45, 61, 59 y 78). CIMAR 3 (St.: 4, 5, 10, 18, 19, 24, 30, 42, 49, 50 (3), 52 (4), 53, 54 y 57). CIMAR 12 FIORDOS (St.: 5, 6, 7, 14, 16, 33, LY2, QL5 y QL9). CIMAR 13 FIORDOS (St.: 36, 37, 39, 43, 84, 85, 86, 82, 76 y 46). CIMAR 14 FIORDOS (St.: 90, 93 y 87). Se le encuentra restringida a las zonas oceánicas de canales y fiordos y rara vez penetra al interior de ellos. No ha sido citada para latidudes más bajas.

Profundidad: entre 5 y 970 m

Globocassidulina subglobosa (Brady, 1881)

(Fig. 3 B; Fig. 4: 6a y b)

Sin.: Cassidulina subglobosa subglobosa Brady 1881, 21: 60; Brady 1884: 9: 430, Pl. 54, fig. $17 \mathrm{a}-\mathrm{c}$.

Cassidulina subglobosa Brady Heron -Allen \& Earland 1932: 359. Earland 1933: 104. Earland 1934: 136, Pl. 6. Figs. 21-22. Barker 1960: 112, Pl 54, Fig. 17. Ingle et al. 1980: 117, Pl. 1, Figs. $14-15$.

Globocassidulina subglobosa Brady. Boltovskoy 1964: 209, P. 18, Fig. 7 a y b. Fillon 1974: 135. Ward \& Webb 1986: 198, Pl 4. Fig. 10. Mateu 1989: 105 y 109, Pl. 6. Fig. 7. Cimerman \& Langer 1991: 63 Figs 4-6. Hromic 1996: 79, Pl. 3, Fig. 1. Cusminsky 1992, 24 (1): 21. Lám. 5, Fig. 1. Murray 1991: 334. Violanti et al. 2000, 521 y 524. Zapata \& Cea 2004: 27, Pl. 8. Fig. 10 y 11.

Diagnosis: Caparazón globoso, esferoidal, ligeramente comprimido con lados desiguales. Paredes lisas con poros muy pequeños, cámaras dispuestas biserialmente, alternadas de manera irregular. Suturas deprimidas. Apertura oblicua a levemente vertical, casi perpendicular al borde, formando una curva, la última cámara envuelve la apertura. Con un reborde en el margen de la última cámara. Paredes lisas, suavemente perforadas.

Observaciones: C. subglobosa es un foraminífero dominante de aguas profundas en todos los océanos y se encuentra entre las especies del 

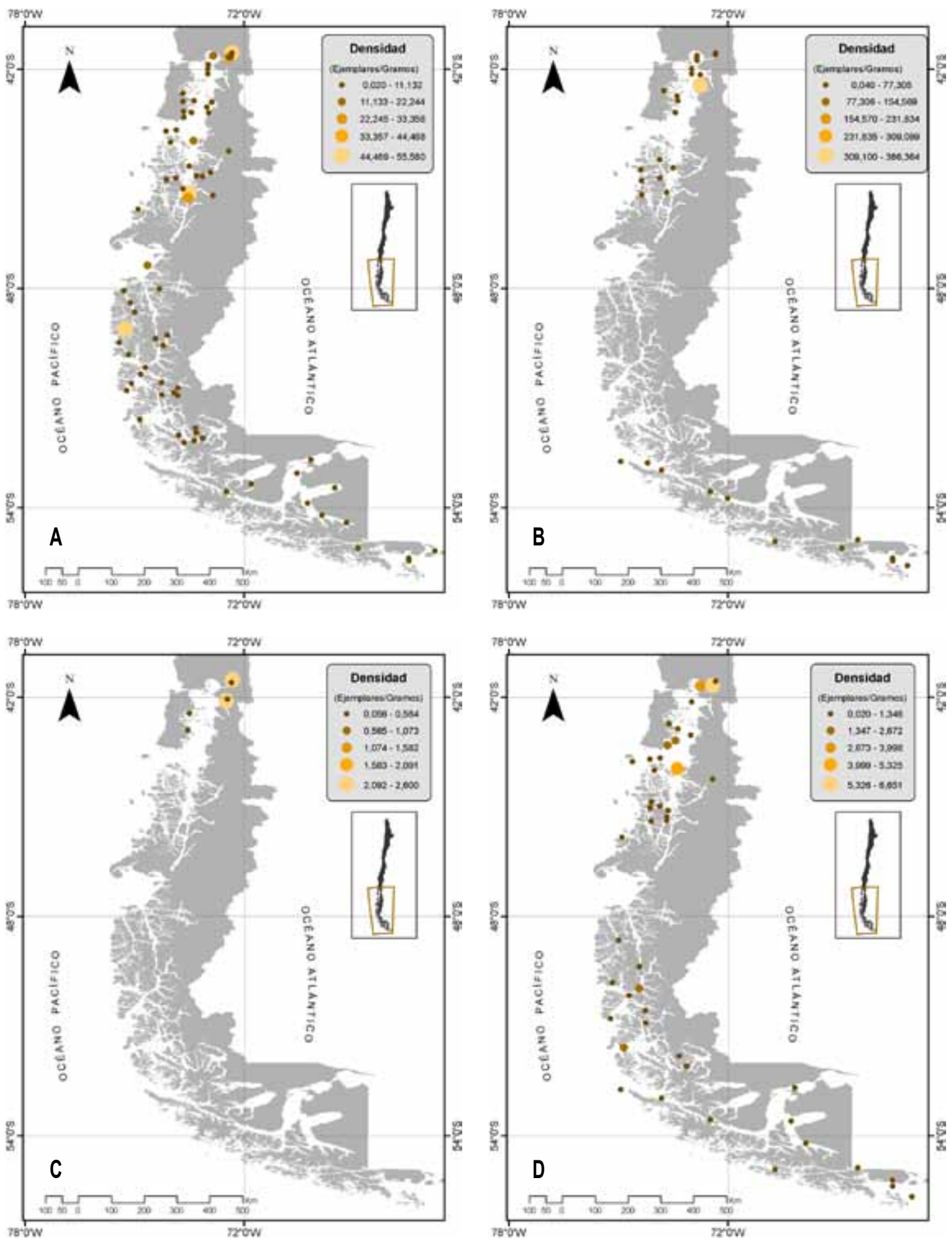

Fig. 3. Distribución geográfica y densidad en canales y fiordos patagónicos de: A.- Globocassidulina rossensis; B.- Globocassidulina subglobosa; C.- Globocassidulina sp. 1, y D.- Ehrenbergina pupa. 
sudeste del océano índico (Corliss 1979). Destaca por su caparazón subglobular (Zapata \& Cea 2004) Abundancia: 449,36 ejemplares / gramo

Distribución latitudinal

Rango: $41^{\circ} 33^{\prime}-55^{\circ} 35^{\prime}$.

Se encontró en 31 muestras: CIMAR 10 FIORDOS (St.: 2, 6, 7, 8, 14, 16, 17, 22, 28, 30, 35 y 39). CIMAR 8 FIORDOS (St.: 49, 65, 9, 54, 63, 14 y 59). CIMAR 3 FIORDOS (St.: 9, 10, 12, 13, 15, 18, 19, 30, 32, 36, 40 y 50.

Profundidad: entre $40 \mathrm{~m}$ y $604 \mathrm{~m}$.

Globocassidulina sp. 1

(Fig. 3 C; Fig. 4: 7)

Diagnosis: Caparazón casi circular, inflado, pared lisa finamente perforada, con suturas definidas, levemente curvadas. La apertura es pequeña, forma una hendidura angosta, paralela al margen periférico, con un labio fino bien desarrollado.

Abundancia: 5,69 ejemplares /gramo.

Distribución latitudinal

Rango: $41^{\circ} 31^{\prime}-42^{\circ} 54^{\prime}$.

Se encontró en 6 muestras: CIMAR 11 (St.: 6, 7, CQ4, HC5, HC6 y DQ4).

Profundidad: entre 108 y 676 m

\section{Género Ehrenbergina Reuss 1850}

Ehrenbergina pupa (d' Orbigny, 1839)

(Fig. 3 D; Fig. 5: $5 \mathrm{a}$ y b)

Sin.: Cassidulina pupa d'Orbigny, 1839 b: 57, lám. 7, figs. 21 - 23.

Ehrenbergina pupa (d'Orbigny) Brady 1884: 433, Pl. 55, fig.1 a y b. Heron - Allen \& Earland 1932: 359, lám. 9, figs. 40 - 47. Earland 1933: 105. Boltovskoy \& Theyer 1970: 326, pl. 2, fig. 24. Zapata \& Varela 1975: 16, pl. 1, figs. 11 a y b. Boltovskoy et al. 1980: 28, pl. 12, figs .13 - 15 . Murray 1991: 331. Zapata et al. 1995: 26, lám. 4, figs. 3 - 5. Hromic 1996: 79. Violanti et al. 2000: 524, pl. 1, fig. 5. Zapata \& Olivares 2000: 56. Cusminsky 1992, 24 (1): 21, lám. 4, fig. 18. Zapata \& Cea 2004: 24, pl. 6, fig. 4.

Diagnosis: Caparazón alargado, enrollado en las primera cámaras, con la sección transversal con forma de triángulo y desenrolladas en las últimas vueltas y dispuestas biserialmente. Bordes romos. Cámaras bajas y anchas, de dos a cinco pares en la sección desenrollada. Paredes lisas, levemen- te perforadas. Suturas curvas, nítidas, limbadas. Apertura en el lado dorsal, con un labio formando una media luna.

Abundancia: 33,11 ejemplares/gramo.

Distribución latitudinal

Rango: $41^{\circ} 33^{\prime}-55^{\circ} 40^{\prime}$.

Se encontró en 49 muestras: CIMAR 11 FIORDOS (St.: 36). CIMAR 2 FIORDOS (St.: 89, $83,43,47,24,40,76,70,56,76 b$ y 57$)$. CIMAR 10 FIORDOS (St.: 35, 39 y 49). CIMAR 8 FIORDOS (St.: 4, 56, 67, 14, 45, 61, 1, 59 y 78). CIMAR 3 FIORDOS (St.: 4, 10, 12, 15, 19, 34, 36, 40, 45, 49, 53 y 55). CIMAR 12 FIORDOS (St.: 3, 5, 7, 16, 36, LY3 y QL9). CIMAR 13 FIORDOS (St.: 36, 37, 41 y 43). CIMAR 14 FIORDOS (St.: 35 y 95).

Profundidad: entre $5 \mathrm{~m}$ y $900 \mathrm{~m}$.

\section{DISCUSIÓN}

El género Cassidulina se encuentra representado en los canales y fiordos patagónicos por dos especies: C. carinata y C. laevigata. Este género presenta una amplia distribución geográfica. Phleger \& Parker (1951) mencionan la presencia de $C$. laevigata en el golfo de México y el Atlántico en playas arenosas hasta $2.870 \mathrm{~m}$, siendo más frecuente entre 90 y 300 m. Ha sido recolectada desde el Ártico (Cushman 1948) hasta la Antártica, entre 658 y 2.450 m (Murray 2002), en especial, en la península antártica (Mackensen \& Hald 1988). C. carinata, ha sido encontrada en la India occidental, al Norte de los $34^{\circ} \mathrm{N}$, entre 102- 612 m (Murray 2002). Es probablemente una especie de antigua data, puesto que se le ha encontrado en sedimentos del Mioceno-Plioceno de Florida, Mioceno de Carolina del Norte, Virginia, Louisiana, California y Jamaica (Cushman \& Todd 1945). Smart (2002), señala que los representantes del género Cassidulina se encuentran en ambientes oxígeno deprimidos y de baja productividad, especificando que $C$. laevigata es una especie que parece preferir aguas frías y de salinidades marinas normales.

En Chile, C. carinata fue recolectada entre los $33^{\circ}$ y $39^{\circ} \mathrm{S}$ en la Fosa Peruano - chilena, $\left(33^{\circ}\right.$ $\mathrm{S}$ Valparaíso; $36^{\circ} \mathrm{S}$ Cabo Carranza y $39^{\circ} \mathrm{S}$ Valdivia), en el batial medio - superior (Ingle et al. 1980). Posteriomente se extendió su rango hacia el norte hasta los $18^{\circ} 28^{\prime}$ (Zapata \& Cea 2004) y hacia el sur, hasta los $47^{\circ} \mathrm{S}$, Canal Baker, estación B3, en 


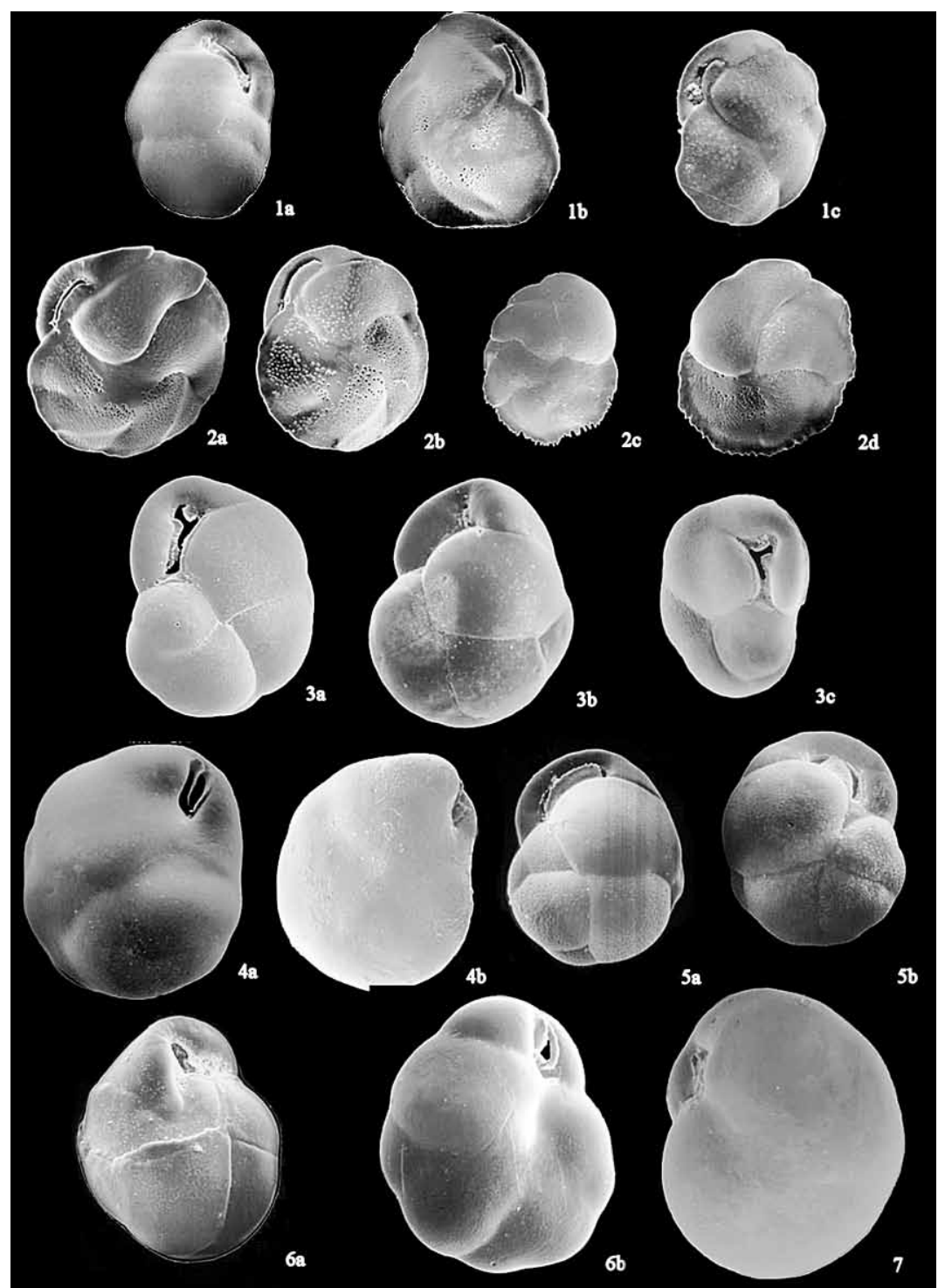

Fig. 4. 1: Cassidulina laevigata a) x 260 , b) x 250 y c) x 400; 2: Cassidulina carinata a) x 210, b) x 195 , c) x 200 y d) x 190 ; 3: Globocassidulina rossensis a) x 240, b) x 260 y c) x 200; 4: Globocassidulina crassa a) x100 y b) x 112; 5: Globocassidulina minuta a) x 240 y b) x 250; 6: Globocassidulina subglobosa a) x 280 y b) x 265 y 7: Globocassidulina sp. 1 × 200.

donde llega a constituir el 22,7\% de los ejemplares (Hromic 2001). Su presencia en estaciones al sur de los $47^{\circ}$ corresponde a nuevos registros. Sus mayores abundancias se observan los canales más septentrionales.

C. laevigata, además de quintuplicar en número de ejemplares, presenta una distribución más amplia tanto latitudinal como en profundidad que carinata. Fue recolectada en el Cabo de Hornos (St. 388) a $121 \mathrm{~m}$ de profundidad (Heron - Allen \&
Earland 1932). Posteriomente, en la zona central chilena (estaciones 96-A, 111-A x1-F, 2957'- 4215'S) (Boltovskoy \& Theyer 1970). (Boltovskoy \& Totah 1987) ajustaron su rango de distribución entre $23^{\circ} \mathrm{S}$ y $46^{\circ} \mathrm{S}$, señalando abundancias escasas de 0,5 $\%$ en la estación 3-9 $\left(23^{\circ} \mathrm{S}\right), 1,9 \%$ en la estación 4-3 (46 $\mathrm{S})$ y del 2,3\%, en la estación 5-3 (45 S). C. laevigata se recuperó también en aguas más profundas, en la Fosa Peruano - chilena, $\left(33^{\circ} \mathrm{S}\right.$ Valparaíso; $36^{\circ} \mathrm{S}$ Cabo Carranza y $39^{\circ} \mathrm{S}$ Valdivia), 


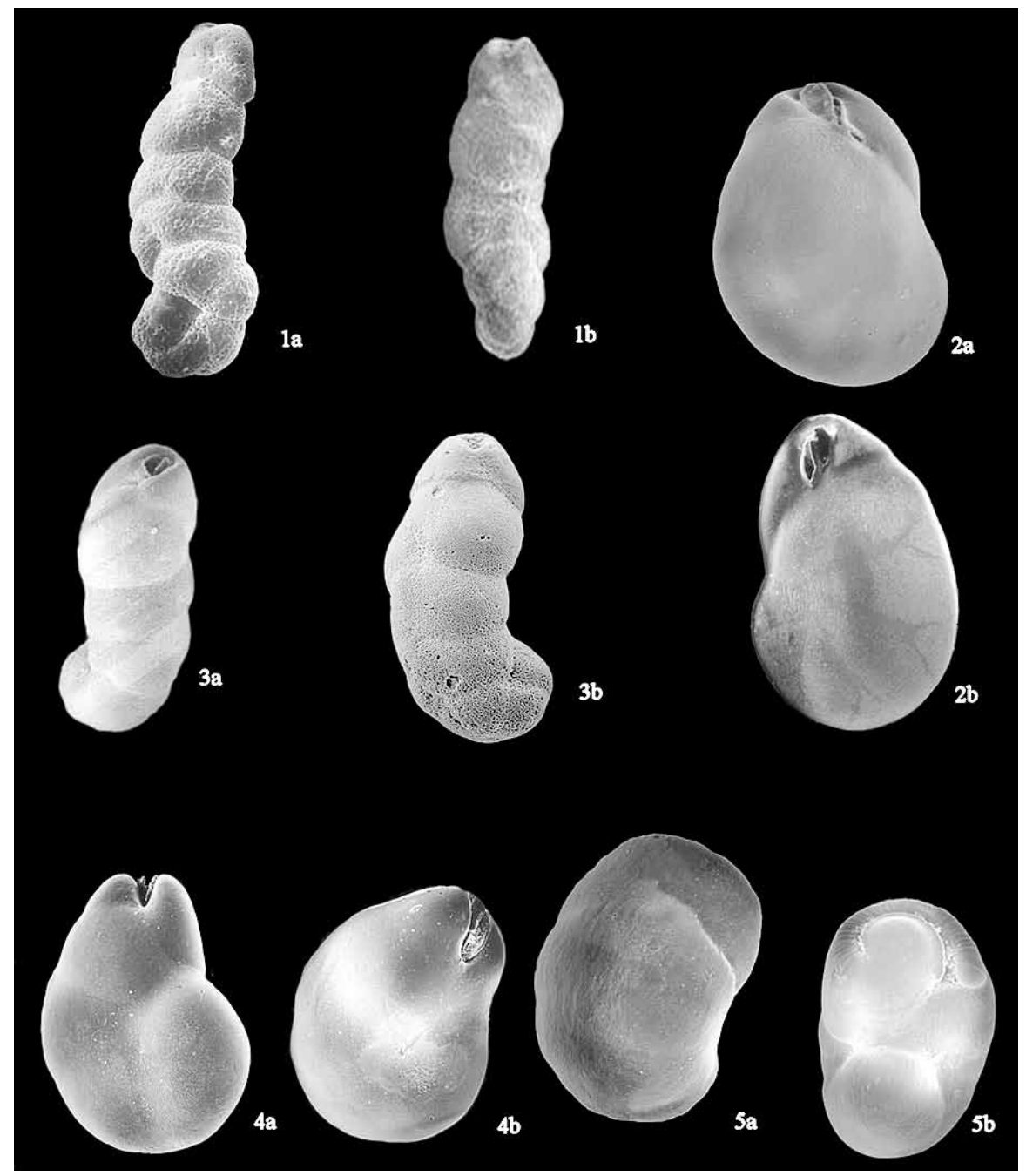

Fig. 5. 1: Cassidulinoides parvus a) x 210 y b) x 195; 2: Cassidulinoides bradyi a) x 180 y b) x 160; 3: Cassidulinoides parkerianus a) x 150 y b) x 145; 4: Cassidulinoides porrectus a) x 90 y b) x 90 y 5: Ehrenbergina pupa a) x 195 y b) x 200 .

en el batial superior (Ingle at al. 1980) coexistiendo con carinata. Zapata et al. (1995), la registran en sedimentos someros, al sur de Chile entre Valdivia y Puerto Montt (40 - $42^{\circ}$ S) y Zapata \& Moyano (1996) entre $39^{\circ}-41^{\circ} 52^{\prime} \mathrm{S}$ (0 -20 m). Hromic (1996) menciona su presncia en el Estrecho de Magallanes, con una representación del 3\%. Zapata \& Moyano (1997) la obtienen nuevamente en el Chile austral entre $41^{\circ} 30 '-53^{\circ} \mathrm{S}$, a profundidades menores de $50 \mathrm{~m}$ en las estaciones $\mathrm{N}^{\circ}$ 1, 2, 13, 1417 y 18 . Zapata \& Cea (2004) extienden su rango por el norte hasta los $18^{\circ} 28^{\prime} \mathrm{S}-31^{\circ} 56^{\prime} \mathrm{S}$. La última cita corresponde a Figueroa et al. (2005), quienes la encuentran en la costa chilena entre $36^{\circ}$ y $44^{\circ}$
S), en las estaciones $\mathrm{N}^{\circ} 1,2,3,4,5,6,11,12,13$, 15, 16,17, 19, 20, 21, 22, 23, 24, 25, 26, 28 y 29. Cusminsky (1992) comunica que C. laevigata llega hasta el Atlántico. Heron- Allen \& Earland (1932) la encuentran en las Islas Falkland, en 28 estaciones, con escasos ejemplares, siendo más comunes al norte de las Islas. Según (Boltovskoy et al. 1980) se extiende desde el Cabo de Hornos hasta aproximadamente los $49^{\circ} \mathrm{S}$ por la costa Atlántica (San Julián) y su presencia la relaciona con la corriente del Cabo de Hornos. Destaca el hecho de que en canales y fiordos esta especie logra internarse en los fiordos conviviendo con especies de caparazón arenáceo (Hromic et al. 2006). Sus mayores 
abundancias se dan entre $41-48^{\circ} \mathrm{S}$. Esta especie puede considerarse cosmopolita, habitando desde los $18^{\circ} \mathrm{S}$ en la costa chilena hasta los $49^{\circ} \mathrm{S}$ en la costa Atlántica, extendiéndose hasta las Falkland, independiente de la profundidad.

En relación a Cassidulinoides bradyi, esta especie ha sido citada sólo en la fosa Peruano- Chilena. Ingle et al. (1980) mencionan su presencia entre $33^{\circ}$ y $39^{\circ}\left(33^{\circ} \mathrm{S}\right.$ Valparaíso; $36^{\circ} \mathrm{S}$ Cabo Carranza y $39^{\circ}$ $S$ Valdivia), en el batial medio, superior e inferior. En este trabajo se comunica el primer registro para la zona de canales y fiordos, habita de preferencia en la zona austral de estos. Se amplía el rango de distribución en aguas chilenas.

Cassidulinoides parkerianus, es la especie más abundante del género y fue registrado inicialmente en el Cabo de Hornos, (St. 388) a $121 \mathrm{~m}$ de profundidad (Heron - Allen \& Earland 1932). Boltovskoy \& Theyer (1970) ampliaron su registro hasta la zona central (estaciones $1 \mathrm{R}$; $96 \mathrm{R}, \mathrm{X} 1 \mathrm{~A}$; X2 A, $\left.29^{\circ}-42^{\circ} \mathrm{S}\right)$ y Boltovskoy \& Totah (1987) mencionan 2 ejemplares en la estación 5 - 4 (48,52 S). C. parkerianus fue encontrado también en Bahía Scholl (52 $\left.44^{\prime} \mathrm{S}\right)$ a $18 \mathrm{~m}$, con una abundancia relativa del 0,19\% (Marchant 1993); entre $39^{\circ}-41^{\circ}, 52^{\prime} \mathrm{S}$ a profundidades < $20 \mathrm{~m}$ (Zapata et al. 1995) y en el Estrecho de Magallanes (Zapata \& Alarcón 1988). Esta especie es nuevamente recolectada entre Valdivia y Puerto Montt $\left(40^{\circ}-42^{\circ}\right.$ S) (Zapata \& Moyano 1996), en el Chile austral, entre $41,30^{\circ}-53^{\circ} \mathrm{S}$, a profundidades $<50 \mathrm{~m}$, en las estaciones $\mathrm{N}^{\circ} 2,3$, 16, 17, 18, 22 y 23) (Zapata \& Moyano 1997) y entre $36^{\circ}-44^{\circ} \mathrm{S}$, en la estaciones $\mathrm{N}^{\circ} 14,20,22$, 23 y 26 (Figueroa et al. 2005). En el norte de Chile se recolectó entre $18^{\circ}, 28^{\prime}-31^{\circ} 56^{\prime} \mathrm{S}$ (Zapata \& Cea 2004). En la región continental austral, fuera del territorio chileno $C$. parkerianus fue citado en Isla de los Estados, en 16 estaciones (Thompson 1978) y en Ushuaia, en donde se encontraron abundantes caparazones vacíos, a menos de $9 \mathrm{~m}$ de profundidad (Lena 1967). C. parkerianus alcanza hasta los $35^{\circ} \mathrm{S}$ en la costa atlántica y se presenta en las islas Malvinas (Boltovskoy et al. 1980) Presenta una distribución similar a C. laevigata. Alcanza su mayor representación en la parte norte de la zona de canales y fiordos.

Respecto de Cassidulinoides porrectus, hay escasa información. Esta especie fue recolectada en 11 estaciones, en el sector de las Falkland ( $\mathrm{He}$ - ron- Allen \& Earland 1932). En la costa atlántica se extiende desde el Cabo de Hornos hasta ca. los $49^{\circ}$ S, San Julián (Boltovskoy et al. 1980). Este mismo autor señala que entre crassa y porrectus se observa un conjunto gradual de formas intermedias. Murray (2002) señala que C. porrectus ha sido registrado en el mar de Ross entre $164 \mathrm{y}$ $475 \mathrm{~m}$, mientras que parkerianus aunque es raro en Antártica, se le encuentra entre 365 y 1.670 m. Es la primera cita para aguas chilenas, mostrando las mayores abundancias en la parte central y norte de los canales y fiordos.

Cassidulinoides parvus se registró en el Cabo de Hornos (St. 388) a $121 \mathrm{~m}$ de profundidad, con una alta abundancia y forma muy típica (Heron- Allen \& Earland 1932). Posteriormente se registró en el Chile austral entre $41,30^{\circ}$ y $53^{\circ} \mathrm{S}$, a menos de 50 $m$ de profundidad, en las estaciones $\mathrm{N}^{\circ} 17,20$ y 25 (Zapata \& Moyano 1997). Esta especie se presenta claramente en los canales más meridionales. Se amplía su distribución hasta los $55^{\circ} 40^{\prime} \mathrm{S}$.

El género Globocassidulina estuvo representado por cuatro especies: G. crassa, G. minuta, G. rossensis y Globocassidulina sp. 1. Smart (2002) señala que al contrario de Cassidulina, el género Globocassidulina (G. subglobosa), es abundante en ambientes con alta disponibilidad de oxígeno.

Globocassidulina crassa, es una especie que se presenta con una distribución más bien restringida. Phleger \& Parker (1951) señalan que es difícil determinar los caracteres exactos de crassa a partir de la figura de d'Orbigny y que es probable que los ejemplares ilustrados por este autor correspondan a dos especies diferentes. De igual manera, se hace referencia a que G. subglobosa ha sido confundida con crassa por muchos autores, Para este trabajo se ha seguido la identificación de Brady (1884) y la revisión efectuada por Barker (1960).

En Chile, G. crassa se ha encontrado desde el Cabo de Hornos (St. 388), a 121 m de profundidad, (Heron- Allen \& Earland 1932) hasta Arica, en la zona norte de Chile $\left(18^{\circ}-21^{\circ} \mathrm{S}\right)$ (Coulbourn 1980). Su presencia está documentada a lo largo de la costa chilena, a saber: Páez et al. (2001), en bahía Mejillones ( $\left.23^{\circ} \mathrm{S}\right), 3,4 \%$ de los ejemplares, a $56 \mathrm{~m}$ de profundidad, en sedimentos micróxicos; Boltovskoy \& Theyer (1970) en la zona central de Chile (Estaciones 96F 109 F, 111 ${ }^{\mathrm{a}}$, X1A y X2 R: $29^{\circ}-42^{\circ}$ S); Boltovskoy \& Totah (1987) entre $23^{\circ}$ 
y $46^{\circ} \mathrm{S}$, en la estaciones $3-9\left(23^{\circ} \mathrm{S}\right), 4-3\left(46^{\circ} \mathrm{S}\right)$ y $5-3\left(45^{\circ} \mathrm{S}\right)$ con una abundancia de $0,5 \%, 1,9 \% \mathrm{y}$ $2,3 \%$ respectivamente y Figueroa et al. (2005) entre $23^{\circ} \mathrm{S}-44^{\circ} \mathrm{S}$ en la estaciones $\mathrm{N}^{\circ} 10,11,13,14,15$, 17, 20, 21, 22, 23, 24, 25, 28 y 29);

Zapata et al. (1995), la registraron en el sur de Chile, (39 $\left.-41^{\circ} \mathrm{S}\right)$ a menos de 20 m profundidad); Zapata \& Moyano (1996), entre Valdivia y Puerto Montt (40 - $\left.42^{\circ} \mathrm{S}\right)$; Zapata \& Varela (1975) en ba-

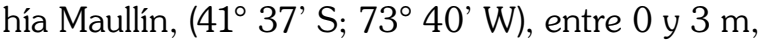
señalando que los ejemplares son más pequeños 0,30 - 0,33 mm de diámetro, que los encontrados por d'Orbigny en el Cabo de Hornos; Zapata \& Alarcón (1988) en el Estrecho de Magallanes; Zapata \& Moyano (1997), en Chile austral $\left(41,30^{\circ}-53^{\circ} \mathrm{S}\right)$ en las estaciones $\mathrm{N}^{\circ} 1,7,8,14-18,22$ y 28 , a menos de $50 \mathrm{~m}$ de profundidad.

Fuera del Chile continental se cita su presencia en el archipiélago de Juan Fernández, aunque con escasos ejemplares (Cushman \& Wickenden 1929). Años más tarde, Zapata (1999) encuentra entre 4 y 15 ejemplares en bahía Cumberland (J. Fernández), a menos de $12 \mathrm{~m}$ de profundidad.

Globocassidulina crassa se registró en forma abundante en Ushuaia, canal Beagle. Citada como Cassidulina crassa d'Orbigny forma intermedia, se pone en sinonimia con $C$. crassa citada por Boltovskoy \& Theyer (1970). Se describe como especie característica de la corriente de las Malvinas (Lena 1967). Boltovskoy et al. (1980) señalan que Cassidulina crassa f. typica llega por el atlántico hasta ca. $49^{\circ} \mathrm{S}$, (Puerto San Julián). Thompson (1978) la encontró en la Isla de los Estados, (Atlántico Sudoccidental) en 40 estaciones, en áreas abiertas, protegidas, bahías suaves y fiordos, en sedimento lodoso-calcáreo. Destaca que en las estaciones $\mathrm{N}^{\circ}$ 673 y N N $^{\circ} 674$ alcanza una abundancia del 30\%. Heron - Allen \& Earland (1932) informaron que se encuentra presente en las Islas Falkland, con distribución universal (en 40 estaciones). Esta especie se encuentra en los canales con escasa abundancia y más bien restringida a profundidades bajas, con abundancias mayores en la zona norte y sur de los canales, sugiriendo una distribución disyunta. También puede considerarse una especie cosmopolita de plataforma.

Otro representante encontrado en canales y fiordos patagónicos fue Globocassidulina minuta. Cushman (1933) destaca que esta especie, sólo se encuentra en aguas profundas del Pacífico tropical. A diferencia de crassa esta especie predomina en los canales y fiordos aportando el $42 \%$ de los ejemplares de cassidulinoídeos. Ocupa prácticamente toda la zona de canales y fiordos, alcanzando las mayores abundancias en la parte norte. Se ha documentado su presencia en el Estrecho de Magallanes (Zapata \& Alarcón 1988); Bahía Scholl (52 44’ S), 18 $\mathrm{m}, 7 \%$ de los ejemplares (Marchant 1993); entre $41,30^{\circ}$ y $50^{\circ} \mathrm{S}$, Chile austral, a menos de $50 \mathrm{~m}$ de profundidad en la estaciones $\mathrm{N}^{\circ} 19,24,25$ y 28 (Zapata \& Moyano 1997); en canales patagónicos $\left(42^{\circ} \mathrm{S}-47^{\circ} \mathrm{S}\right)$, en al menos una estación (St. 12), con escasa abundancia (6 ejemplares), en aguas someras (7 m) (Hromic \& Zúñiga 2005); en bahía Yendegaia $\left(\sim 55^{\circ} \mathrm{S}\right)$, Canal Beagle, $(0,4 \%)$ (Zúñiga-

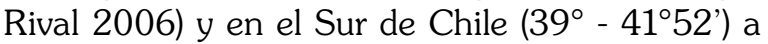
menos de $20 \mathrm{~m}$ de profundidad (Zapata et al. 1995). En la Fosa Peruano - chilena ( $33^{\circ} \mathrm{S}$ Valparaíso; $36^{\circ}$ $S$ Cabo Carranza y $39^{\circ} \mathrm{S}$ Valdivia), en el borde de la plataforma (> $135 \mathrm{~m}$ ) (Ingle et al. 1980). También se recolectó en bahía Cumberland, 5 ejemplares, 8 m, Juan Fernández (Zapata 1999) y en Isla de Pascua (2710' S; $109^{\circ} 20^{\prime} \mathrm{W}$ ) a menos de $5 \mathrm{~m}$ (Zapata \& Olivares 2000). Lena (1967) la recolectó en forma abundante en Ushuaia, a menos de $9 \mathrm{~m}$ de profundidad y señala que se le encuentra en la costa argentina (Río de la Plata). Se distribuye desde los $33^{\circ} \mathrm{S}$ en la costa chilena hasta el río de la Plata incluyendo las islas de las Malvinas.

Otra especie del género Globocassidulina registrada en los canales y fiordos fue $\mathrm{G}$. rossensis. Ishman \& Martínez (1995) encontraron abundantes ejemplares entre $42^{\circ}$ y $55^{\circ} \mathrm{S}$. Hromic et al. (2006) señalan que rossensis es un componente importante en las biofacies oceánicas y de canales intermedios. Más al sur, es citada en bahía Yendegaia $\left(\sim 55^{\circ} \mathrm{S}\right)$, canal Beagle (0,8\%) (Zúñiga-Rival 2006). Boltovskoy et al. (1980) la citan en la costa argentina en Mar del Plata $\left(35^{\circ} \mathrm{S}\right)$ y Tierra del Fuego. En este estudio se presenta en toda la zona de canales y fiordos.

Globocassidulina subglobosa se recolectó en el Cabo de Hornos (St. 388) a 121 m de profundidad (Heron - Allen \& Earland 1932). Hromic (1996) la registró en el Estrecho de Magallanes (28\%) y Zapata \& Moyano (1997) en el Chile austral (41,30 $\left.-53^{\circ} \mathrm{S}\right),<50 \mathrm{~m}$, Sts. 7, 12 y 22. G. subglobosa se presentó en 11 estaciones situadas frente a Arica, zona norte de Chile entre $18^{\circ}-21^{\circ} \mathrm{S}$, con escasos 
ejemplares, $(<3 \%)$ entre 909 y 2.700 m (Coulbourn 1980). También se recuperó desde la Fosa Peruano - chilena, $\left(33^{\circ} \mathrm{S}\right.$ Valparaíso; $36^{\circ} \mathrm{S}$ Cabo Carranza y $39^{\circ} \mathrm{S}$ Valdivia) en el borde de plataforma, batial superior (Ingle et al. 1980). Boltovskoy \& Totah (1987) la encontraron en las estaciones $3-9\left(23^{\circ}\right.$

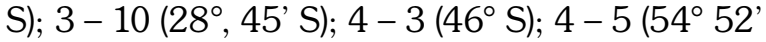
S) y $5-4\left(48^{\circ} 52\right.$ ' S), con abundancias del $2,2 \%$, $0,4 \%, 1 \%, 0,6 \%$ y $23 \%$ respectivamente. También es frecuente en las Malvinas (Sitio 327: 1; 327 A: 1 y 329) (Boltovskoy 1987). Heron- Allen \& Earland (1932) la encuentran en esta misma localidad en 37 estaciones, en menor número que $C$. crassa. Esta especie también se puede clasificar como especie cosmopolita encontrándose tanto en la costa pacífica como atlántica.

Globocassidulina sp 1: se encuentra en los canales de la zona norte patagónica. Es probable que sea un ecotipo de Cassidulina pulchella, sin embargo requiere de un estudio más detallado y por ahora se optó por dejarla con nomenclatura abierta.

Ehrenbergina pupa fue encontrada en el Cabo de Hornos (St. 388), a 121 m de profundidad (Heron - Allen \& Earland 1932). En el Pacífico se le cita en el Estrecho de Magallanes (Hromic 1996); en el Chile austral (41,30 ${ }^{\circ}$ y $\left.53^{\circ} \mathrm{S}\right),<50 \mathrm{~m}$ (Zapata \& Moyano 1997); en aguas someras ( $42^{\circ}$ y $47^{\circ}$ S), Estaciones $\mathrm{N}^{\circ} 11$ y $\mathrm{N}^{\circ} 12$, a $7 \mathrm{~m}$, (Hromic \& Zúñiga 2005); en bahía Yendegaia, canal Beagle (0-7 m) (0,5 \%) (Zúñiga-Rival 2006). En la zona central, estaciones $N^{\circ} 21$ y 22 R, 109a x1 A (29 $\left.-42^{\circ} 15^{\prime} \mathrm{S}\right)$, (Boltovskoy \& Theyer 1970). En bahía Maullín (41 $37^{\prime} \mathrm{S}$; 73 $\left.40^{\prime} \mathrm{W}\right), 0,35$ - 0,40 mm de diámetro, característica de aguas frías (Zapata \& Varela 1975). En Tocopilla (22 ${ }^{\circ} 06^{\prime}$ S) (Zapata \& Gutiérrez 1995). Entre Valdivia y Puerto Montt (40 - 42 ${ }^{\circ}$ S) (Zapata \& Moyano 1996). En la zona norte de Chile $\left(18^{\circ}-21^{\circ} \mathrm{S}\right)$, frente a Arica (estación F62; 30 m) (Coulbourn 1980). En la costa chilena $\left(23^{\circ} \mathrm{S}-44^{\circ} \mathrm{S}\right)$ (estaciones: $15,20,21,22,23,25$, 26 y 29) (Figueroa et al. 2005). Entre $18^{\circ}, 28^{\prime}-31^{\circ}$ 56' S (Zapata \& Cea 2004). En el Atlántico, en Isla de los Estados, en 10 estaciones (Thompson 1978). En forma abundante en 30 estaciones de las islas Malvinas (Heron- Allen \& Earland 1932). Murray (2002) indica que Ehrenbergina, está presente entre 20 a $200 \mathrm{~m}$. Esta especie si bien es conocida a lo largo de la costa chilena presenta sus mayores abundancias en la zona de los canales.

\section{CONCLUSIONES}

En canales y fiordos la Familia Cassidulinidae se encuentra representada por doce especies, siendo las más abundantes: Globocassidulina minuta (42,2\%), Globocassidulina subglobosa (19,9\%) y Globocassidulina rossensis $(16,6 \%$ ) y las con mayor distribución geográfica, Globocassidulina rossensis $(50,6 \%$ de las muestras), Cassidulinoides parkerianus $(42,1 \%)$, Cassidulina laevigata $(34,8 \%)$ y Ehrenbergina pupa (29,9\%). De las especies recuperadas en los canales solo tres se restringen a ellos: Cassidulinoides parvus, Cassidulinoides porrectus y Globocassidulina rossensis. G. minuta y $C$. bradyi se registran hasta los $33^{\circ} \mathrm{S}$ y el resto ha sido mencionado en aguas del norte del país.

\section{AGRADECIMIENTOS}

Los autores agradecen al Comité Oceanográfico Nacional (CONA) Chile, el financiamiento de los proyectos que respaldan esta publicación. A la Universidad de Magallanes por el apoyo brindado para la ejecución de los proyectos. A la Sra. Tatiana Hromic, del Instituto de la Patagonia, Investigadora responsable de los proyectos CIMAR, quien cedió las muestras para este estudio y por su paciencia y buena voluntad para orientar este trabajo.

\section{LITERATURA CITADA}

Barker, R.W. 1960. Taxonomic Notes on the Species figured by H. B. Brady in his Report on the Foraminifera dredged by H.M. S. Challenger during the years 1873-1876. Society Economical, Paleontological \& Mineral, Special Pub. № 9, 238 pp.

Boltovskoy, E. 1964. Provincias zoogeográficas de América del Sur y su sector antártico según los foraminíferos bentónicos. Boletín del Instituto de Biología Marina, 7:93-98

Boltovskoy, E. 1965. Foraminíferos recientes. Eudeba, 510 pp.

Boltovskoy, E. \& F. Theyer 1970. Foraminíferos Recientes de Chile Central. Revista Museo Argentino de Ciencias Naturales "Bernandino Rivadavia" e Instituto Nacional de Investigación de las Ciencias Naturales, Hidrobiología, 2(9):279-379 
Boltovskoy, E \& V. Totah 1987. Relación entre masas de agua y foraminíferos bentónicos en el Pacífico sudoriental. Physis, Secc. A. 45(109):37-46

Boltovskoy, E., G. Giussani, S. Watanabe \& R. Wright 1980. Atlas of benthic shelf foraminifera of the southwest Atlantic Junk by Pub. The Hague - Boston - London. $147 \mathrm{pp}$.

Brady, H.B. 1881. Notes on some of the reticularian Rhizopoda of the "Challenger" Expedition; Part III. Quarterly Journal of the Microscopical Science, 21:31-71

Brady, H.B. 1884. Report on the Foraminifera dredged by H.M.S. "Challenger" during the years 1873-1876. Report Voyage Challenger, Zoology, 9:814 pp.

Cimerman, F. \& Langer M. 1991. Mediterranean Foraminifera. Paleontoloski Institut Ivana Rakovca, 118 pp. 93 Figs.

Corliss, B. 1979. Size variation in the deep sea benthonic foraminifer Globocassidulina subglobosa (Brady) in the southeast Indian Ocean. Journal Foraminiferal Research, 9(1):50-60

Coulbourn, W. 1980. Relationship between the distribution of Foraminifera and geologic structures of the Arica Bight, South America. Journal of Paleontology, 54(4):696-718

Cushman, J. 1922. The Foraminifera of the Atlantic Ocean Part 3- Textulariidae U.S. Nat. Museum, Bulletin, 49pp.

Cushman, J. \& D. Hugues 1925. Some later tertiary Cassidulina of California. Contribution from Cushman Laboratory for Foraminiferal Research, 1(1):11-17

Cushman, J. \& F. Parker 1931. Miocene Foraminifera from the Temblor of the East Side of the San Joaquín Walley, California. Contribution from Cushman Laboratory for Foraminiferal Research, 1-16

Cushman, J. 1933. Some new recent foraminifera from the tropical pacific. Contribution Cushman Laboratory for Foraminiferal Research, 9(4):77-95

Cushman, J. \& R. Wickenden 1929. Recent foraminifera from off Juan Fernández Islands. Procceeding United States Natural Museum, 75(9):1-16

Cushman, J. \& R. Todd 1945. Miocene foraminifera from Buff Bay, Jamaica. Contribution Cush- man Laboratory for Foraminiferal Research, Special. Pub.: 15:1-75

Cushman, J. 1948. Arctic foraminifera. Contribution Cushman Laboratory for Foraminiferal Research, Special Pub. 23. 70 pp; 8 Lám.

Cusminsky, G. 1992, Foraminíferos Bentónicos del Océano Atlántico Sudoccidental Austral. Revista Española de Micropaleontología, 24(1):18-30, Lám. 5

Decrouez, D. 1989. Generic ranges of Foraminiferida. Revue Paleobiologie, 8(1):263-321

Earland, A. 1933. Foraminifera. Part II. South Geogia. Discovery Report, 7(1):27-138. Pl. I - VII.

Earland, A. 1934. Foraminifera Part III: The Falklands sector of the Antartic (excluding South Georgia). Discovery Report. X: 1-208. Pl. I - X

Ellis, B. \& A. Messina 1940. (et seq.) Catalogue of Foraminifera. American Museum of Natural History. Special Pub. 30 vol.

Fillon, R.H. 1974. Late Cenozoic foraminiferal paleoecology of the Ross Sea, Antarctíca. Micropaleontology, 20:129-151

Finger, K.L. \& J.H. Lipps 1981. Foraminiferal decimation and repopulation in an active volcanic caldera Deception Island, Antarctica. Micropaleontology, 27(2):111-139

Figueroa, S., M. Marchant, S. Giglio \& M. Ramírez 2005. Foraminíferos bentónicos rotalínidos del centro-sur de Chile $\left(36^{\circ} \mathrm{S}-44^{\circ} \mathrm{S}\right)$ Gayana Zoología, 69(2):329-363

Heron - Allen, E. \& A. Earland 1932. Foraminifera. Pt. 1. The ice-free area of the Falkland Islands and adjacent watter. Discovery Report, 4:291-460

Hromic, T. 1991. Foraminíferos de la Formación Brush Lake: Cuenca Austral, Chile. Anales Instituto Patagonia Serie Cs. Nat. (Chile), 20:101-111

Hromic, T. 1996. Foraminíferos bentónicos (Protozoa: Foraminiferida) de aguas profundas del estrecho de Magallanes, Chile. Anales Instituto Patagonia Serie Cs. Nat. (Chile), 24:65-86

Hromic, T. 2001. Foraminíferos bentónicos del canal Baker (47은 $\mathrm{S}$; $4^{\circ} \mathrm{W}$ ), Pacífico sudoriental, Chile. Anales Instituto Patagonia, Serie Cs. Nat. (Chile), 29:135-156

Hromic, T. 2009 a. Estructura comunitaria del taxa Foraminiferida (Protozoa) según profundidad, 
en el fiordo Comau, Chiloé, Anales Instituto Patagonia Serie Cs. Nat. (Chile), 37(1):39-51 Hromic, T. 2009 b. Distribución batimétrica de foraminíferos bentónicos (Protozoa: Foraminiferida) al sur del estrecho de Magallanes (52으 - 56음 S), Chile. Anales Instituto Patagonia (Chile), 37(1):23-38

Hromic, T. 2011 a. Foraminíferos bentónicos recolectados durante la expedición Cimar 14 Fiordos, Patagonia Chilena. Anales Instituto Patagonia (Chile), 39(1):75-89

Hromic, T. 2011 b. Foraminíferos bentónicos de la expedición Cimar 11 Fiordos, canales patagónicos chilenos: biodiversidad y abundancia. Ciencia y Tecnología del Mar, 39(1) (en prensa)

Hromic, T. 2011 c. Análisis de la comunidad foraminiferológica bentónica del seno Reloncaví, Islas Desertores, golfo Ancud y golfo Corcorvado, Chile. Ciencia y Tecnología del Mar, (en prensa)

Hromic, T \& M. Zúñiga 2005. Foraminíferos bentónicos de ambientes someros extraídos durante la expedición CIMAR 7 FIORDOS, Canales patagónicos (42으 - 47오). Boletín de la Sociedad de Biología de Concepción, 76:25-38

Hromic, T., S. Ishman \& N. Silva 2006. Benthic foraminiferal distribution in Chilean fjords: $47^{\circ} \mathrm{S}$ to $54^{\circ} \mathrm{S}$ Marine Micropaleontology, 59:115-134

Ingle, J., G. Keller \& R. Kolpack 1980. Benthic foraminiferal biofacies sediments and water masses of the southern Perú-Chile Trench area southeastern Pacific Ocean. Micropaleontology, 26(2):113-150

Instituto Geográfico Militar, 1993. Gran Atlas de Chile y el Universo. Ed. Occidente, 102 pp.

Ishman, S. \& R. Martínez 1995. Distribution of modern Benthic Foraminifers from fjord región of southern Chile ( $42^{\circ} \mathrm{S}$ to $55^{\circ} \mathrm{S}$ ) Antarctic Journal Review, 6 - 8

Lena, H. 1967. Foraminíferos recientes de Ushuaia (Tierra del Fuego, Argentina). Ameghiniana, 4(9):311-336

Loeblich, A. \& H. Tappan 1988. Foraminiferal Genera and Their Classifications. Van Nostrand Reinhold Co. Text- Vol.: 970 pp. Pl. - Vol.: 212 pp. +847
Mackensen, A. \& M. Hald 1988. Cassidulina teretis and Cassidulina laevigata D'Orbigny: Their modern and late quaternary distribution in northern seas. Journal of Foraminiferal Research, 18(1):16-24

Marchant, M. 1990. Foraminíferos miocénicos de los estratos de Pupunahue (provincia de Valdivia, $X^{\underline{a}}$ Región); determinación de la edad probable y paleoambiente. II음 Simposio sobre el Terciario de Chile, Actas. Tomo 1:177-188

Marchant, M. 1993. Foraminíferos de la Bahía Scholl, Región Magallánica, Chile, (Protozoa: Foraminifera). Gayana Zoología, 57(1):61-75 Martínez, R. 1976 Hallazgo de foraminíferos miocénicos cerca de Puerto Aldea, bahía de Tongoy, Provincia de Coquimbo, Chile. Revista Geológica de Chile 8:65-78. In: Marchant, M. Bibliografía Micropaleontológica de Chile. Anales Instituto Patagonia. Serie Cs. Nat. (Chile), 1989-1990, 19(1):67-80

Martínez, G.R. \& R. Martínez 1994. Foraminíferos bentónicos recientes de Bahía Chile, Isla Greenwich, Islas Shetland del Sur. Su significado para la historia paleoclimática holocena de la Antártica Occidental. 7ํㅡㄴ Congreso Geológico Chileno. Universidad de Concepción, Chile: 1689-1692. Actas

Martínez, R. \& R. Osorio 1968. Foraminíferos pliocénicos de Chile Central. II. Edad y Paleoecología de la Formación Tubul. In: Cecioni (Ed.) 1968. El Terciario de Chile. Zona central. Sociedad Geológica de Chile Ed. A. Bello: 115-164

Martínez, R. \& C. Parada 1968. Foraminíferos pliocénicos de Chile central II. Foraminíferos pliocénicos de San Sebastián (Laguna de los Patos) Provincia de Santiago. In: Cecioni (Ed.) 1968. El Terciario de Chile. Zona central. Sociedad Geológica de Chile Ed. A. Bello: 167-189

Mateu, G. 1989. Micropaleontología sedimentaria. Informe de resultados de la campaña "Antártida 8611". Publicación Especial del Instituto Español de Oceanografía, 1르 Expedición Científica Pesquera en la Antártida, Secretaría General de Pesca Marítima M. A. P. A. Ministerio Agricultura, Pesca y Alimentación, 2:83-173 
Murray, J.W. 1991. Ecology and paleoecology of benthic foraminifera. Longman Scientific \& Technical, 397 pp.

Murray, J.W. 2002. Environmental applications of deep - sea benthic foraminifera. Quaternary Environmental Micropaleontology. Haslett (Ed.) 5 - 13

Natland, M.E. González, A. Cañón \& M. Ernst 1974. A system of stage for correlation of Magallanes Basin Sediments. Geological Society American Memoir 139. 126 pp.

Orbigny, A.d' 1826. Tableau méthodique de la classe des Céphalopodes. Annals Science Natural, 1:245-314

Orbigny, A.d' 1839. Voyage dans l'Amérique Méridionale. Foraminiféres, 5:1-86

Páez, M. \& O. Zúñiga 2001. Foraminíferos recientes

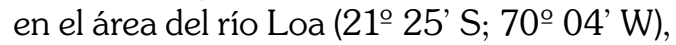
Chile. Estudios Oceanológicos, 20:3-12

Páez, M., O. Zúñiga, J. Valdés \& L. Ortlieb 2001. Foraminíferos bentónicos recientes en sedimentos micróxicos de la bahía Mejillones del Sur (23은 S), Chile. Revista de Biología Marina y Oceanografía, 36(2):129-139

Parker, W. \& T. Jones 1865. On some Foraminifera of the North Atlantic and Arctic Ocean, including Davis straits and Baffin's Bay. Royal Society London, Philosophy Transactions, 155:325-441

Phleger, F \& F. Parker 1951. Ecology of foraminifera, northwest gulf of México. Geology Society American Memoir, 46, Part. II, Foraminiferal species. $125 \mathrm{pp}$.

Smart, C. 2002. Environmental applications of deep - sea benthic foraminifera. Quaternary Environmental Micropaleontology. Haslett (Ed.), 14-58

Tapia, R.C. Lange \& M. Marchant 2008. Living (stained) calcareous benthic foraminifera from recent sediments off Concepción, centralsouthern Chile ( $\left.36^{\circ} \mathrm{S}\right)$. Revista Chilena de Historia Natural, 81:403-416

Thompson, L. 1978. Distribution of living benthic foraminifera, Isla de los Estados, Tierra del Fuego, Argentina. Journal Foraminiferal Research, 8(3):241-257

Violanti, D., B. Loi \& R. Melis 2000. Distribution of recent foraminifera from the Strait of Magellan. First quantitative data. Bolletino
Museo Regional Scientia Nauralli,Torino, 17(2):511-539

Ward, B.L. \& P. Webb 1986. Late Quaternary foraminifera from raised deposits of the Cape Royds-Cape Barne area, Ross Island, Antarctica. Journal of Foraminiferal Research, 16:176-200

Williamson, W. 1858. On the recent Foraminifera of the Great Britain. Royal Society of London (Eds.), 107 pp.

Zapata, J. 1999. Foraminíferos bentónicos recientes

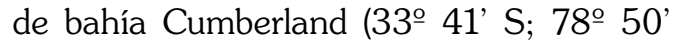
W), Archipiélago de Juan Fernández, Chile: Aspectos zoogeográficos. Boletín de la Sociedad de Biología de Concepción, 70:21-35

Zapata, J.A. \& R. Alarcón 1988. Foraminíferos bentónicos del Estrecho de Magallanes $\left(52^{\circ}\right.$ 33' S; 69 54' W), Chile. Biota, 4:17-29

Zapata, J, \& C. Cea 2004. Foraminíferos litorales recientes del norte de Chile (1828' - 3156' S) y sus relaciones zoogeográficas. Boletín de la Sociedad de Biología de Concepción, 75:7-57

Zapata, J. \& A. Gutiérrez 1995. Foraminíferos litorales recientes de Tocopilla (22응' $\mathrm{S}$; $\left.70^{\circ} 13^{\prime} \mathrm{W}\right)$, Chile. Estudios Oceanológicos, 14:49-59

Zapata, A. \& S. Varela 1975. Foraminíferos litorales

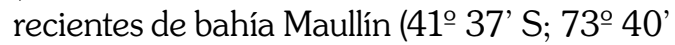
W) Chile. Ciencia y Naturaleza, 16(1):14-24

Zapata, J. \& H. Moyano 1996. Distribución de los foraminíferos bentónicos recolectados por el AKEBONU MARU “72", en el sur de Chile. Gayana Zoología, 60(2):89-98

Zapata, J. \& H. Moyano 1997. Foraminíferos bentónicos recientes de Chile austral. Boletín Sociedad Biología de Concepción, 68:27-37

Zapata, J. \& J. Olivares 2000. Biodiversidad y zoogeografía de los foraminíferos bentónicos de la isla de Pascua (27ำ10' S; 10920'W). Boletín de la Sociedad de Biología de Concepción, 7:53-77

Zapata, J., C. Zapata \& A. Gutiérrez 1995. Foraminíferos bentónicos del sur de Chile. Gayana Zoología, 59(1):23-40

Zúñiga-Rival, M. 2006. Estudio preliminar de los foraminíferos bentónicos (Protozoa: Foraminiferida) de bahía Yendegaia, Tierra del Fuego, Chile. Anales Instituto Patagonia (Chile), 34:33-40 
